\title{
Myoclonic encephalopathy of infants
}

\author{
M. KINSBOURNE \\ From the Hospital for Sick Children, Great Ormond Street, London
}

The intrusion of shock-like, muscular contractions may totally disorganize willed movements involving the affected muscle groups. If the contractions are frequent and violent the appearance may even suggest a cerebellar ataxia. But the persistence of the shock-like contractions when the affected part is at rest, and electroencephalographic evidence of a cerebral dysrhythmia related in its irregular timing to that of the involuntary muscular contractions, reveal the presence of myoclonic status. In most cases in which generalized myoclonic status is present as an isolated abnormality in a child, it proves to be a manifestation either of 'idiopathic epilepsy' or of the onset of a progressive cerebral degeneration.

It is proposed to report six cases in which mycclonic status produced clinical appearances and took a course differing from any previously described and yet conforming to a consistent and distinctive pattern. The acute onset of myoclonic status (especially involving the extraocular muscles in an unusual manner) was unaccompanied by electroencephalographic evidence of cerebral discharges. This isolated motor disorder pursued a protracted but not progressive course.

\section{CASE REPORTS}

CASE 1 S.W., aged 1 year, was admitted under the care of Dr. R. Lightwood after the sudden onset two months earlier, in the course of an upper respiratory tract infection, of continuous jerking of the head and eyes. Within a few days the disorder had reached a plateau of severity which was maintained up to admission. When first seen she was in perpetual motion. Her head, mostly facing to the right, moved incessantly in a rapid, irregular, jerky fashion. Any attempt to maintain the sitting posture was vitiated by jerking affecting the trunk. Intermittent irregular perturbations of the limbs interfered with willed movements; but even at rest muscular twitching was apparent, particularly of the facial muscles. Still more striking was the condition of the eyes. Never more than momentarily at rest, they were continually being displaced, usually, though not always, in a conjugate manner, in all possible directions. Movements of horizontal, vertical, and angular deviation, as well as of rotation followed each other in irregular and unpredictable sequence. The movements, extremely rapid, gave the impression of a continued flickering, or 'dancing', of the eyes. Always of large amplitude, the movements would become still more violent when visual stimuli were applied, or when the child was disturbed or alarmed. They made it impossible for the patient to maintain fixation in any direction.

There was nothing relevant in the family history; S.W. was the product of normal pregnancy and after a natural delivery at term passed an uneventful neonatal period. However, she was definitely behind her elder sister in the rate of acquisition of motor skills, in that at the time of onset of the disorder (when she was 10 months old) she was not yet able to sit securely without support.

There was no abnormality of skull shape or size, and the anterior fontanelle was open. The eye grounds were normal and there were no focal signs of neurological deficit. There was no fever. The blood count, fasting blood sugar level, and serum electrolyte levels were within normal limits. The Mantoux $(1 / 1,000)$ test was negative, and the skull radiograph unremarkable. Electroencephalograms on admission and repeatedly during her protracted period of illness showed no significant abnormality. The spinal and ventricular fluids were normal in pressure and constitution. Lumbar air encephalography as well as ventriculography were negative. A right frontal cortical biopsy, performed when she was 3 years old, and clinically as severely affected as ever, revealed no significant histological abnormalities either in grey or in white matter (Professor Blackwood).

The motor disorder continued a fluctuating course for the next three years. Anticonvulsant treatment was ineffective. During exacerbations there was much dribbling and marked dysphagia. Even in relative remission, involuntary movements were never absent. After three years, however, they gradually cleared, and the patient has now been free from involuntary movement for five years. She remains somewhat clumsy in movement and speech, and intellectually she falls into the educationally subnormal group (I.Q. 60 on the Terman-Merrill scale). In view of the history of slow acquisition of skills in the first year of life (before the onset of the motor disorder) the present intellectual and motor deficit cannot with certainty be attributed to the motor disorder. The long persistence of the disorder, absence of electroencephalographic abnormality, and lack of response to anticonvulsant drugs distinguish it from minor epileptic status, idiopathic or symptomatic. The non-progressive and uncomplicated nature of the disorder, its ultimate spontaneous remission, and the negative cortical biopsy distinguish it from the myoclonic types of progressive cerebral degeneration. 
CASE 2 M.D., aged 17 months, was admitted under the care of Dr. P. R. Evans, having suddenly begun to stagger two days earlier. Within a day he was off his feet altogether, and his eyeballs appeared to flicker rather than maintain fixation. He vomited twice at the onset, but remained fully conscious, alert and afebrile. When first seen he lay on his back with his head turned to the right, and was resentful when displaced from this posture. When he was sat up, large-amplitude jerking of the trunk became evident, and he clutched wildly for support. He was able to grasp objects offered him, but was inaccurate in his movements because these were disturbed by irregular involuntary muscular jerking. Large-amplitude, irregular conjugate jerks of the eyeballs in various directions were incessant. Maximal when he was startled, they were still present in deep sleep, and even beneath the occasionally quivering eyelids the eyeballs were perceptibly still the subject of involuntary movements. Jerking of other parts of the body was not apparent during sleep. At all times the amplitude of the jerks was grossly increased when the boy was startled, excited, or grieved. Though severe in all parts, the jerking appeared particularly to affect the muscles of the neck and trunk. His breathing came in gasps, and he was unable to chew and only just able to swallow. Although he attempted to speak, the result was unintelligible.

There was nothing abnormal in the family history; a normal pregnancy culminated in spontaneous delivery at term. His development paralleled that of his siblings in that he sat unsupported at 6 months and walked at a year; by 11 months he had a vocabulary of more than six words, clearly articulated. There were no relevant previous diseases, and no obvious precipitating causes for the present complaint.

The skull was normal in shape and size, the fundi were normal, and there were no focal neurological signs. There was no fever. Blood count, fasting blood sugar, chest and skull radiographs, and an electroencephalogram were all within normal limits. Serum electrophoresis showed an increased amount of gamma globulin. The cerebrospinal fluid was normal in pressure and composition. Complement-fixation tests against poliomyelitis and influenza viruses were negative.

Treatment with prednisolone (40 mg. per day) effected an initial partial remission, but this was soon followed by relapse. He was discharged; when he was transferred to the care of Dr. P. H. Sandifer three months later the motor disorder was undiminished. Electroencephalography was again negative, but polyelectromyography (Dr. Pampiglione) showed frequent bursts of muscle action potentials in the left triceps, as well as occasional single muscle potentials. An increase in the level of prednisolone dosage had no observable effect on the severity of the motor disorder. Eighteen months later the involuntary movements were still in full swing. Nevertheless, he had become able to feed himself with his fingers, support his head, and creep along the floor. For mechanical reasons his speech remained monosyllabic.

When last seen, this boy's motor disorder had lasted for two years, during which time it remained uncomplicated and at a constant level of severity. Prednisone had given only transitory benefit. The next case report is of a child as severely affected, who obtained complete symptomatic relief on A.C.T.H.

CASE 3 A.M., aged 10 months, was admitted under the care of Dr. R. Lightwood. A month earlier he had developed nasal catarrh and a mild cough and on the same day had received his first injection of triple vaccine. The respiratory tract infection receded, and he remained well until the tenth day following the injection, when he began to hold his head to the right and lost the ability to sit up. His limbs were found to be 'floppy'. From the onset there were rapid irregular movements of the head and unrelated movements of the eyeballs. Over the next few days such movements also caused his limbs to jerk, interfering with their use, and, by involving the muscles subserving swallowing, produced a degree of dysphagia necessitating feeding by tube. Within a week the motor disorder had reached its height; it then remained unchanged for many weeks in spite of treatment with prednisolone (5.0 $\mathrm{mg}$. daily).

On admission he was seriously ill. The intrusion of shock-like muscular contractions disorganized his respiratory movements (which were at first interrupted by periods of apnoea) and vitiated his attempts at moving or swallowing. He lay supine and limply. When his head was lifted from the pillow, titubation became obvious. At rest, irregular twitching was evident, especially in the fingers, feet, and face. The eyeballs made quick conjugate movements of deviation and rotation without pause. The motor disorder was most marked when the child was handled or otherwise disturbed. But it was evident also when he was relaxed, or drifting off into a light sleep, when the jerking movements of the eyeballs could still be detected beneath the closed eyelids. Only in deep sleep was the jerking no longer apparent.

Nevertheless, he very occasionally made volitionake movement smoothly and without irregularity. At these? times the absence of ataxia of cerebellar type was obvious.

The family history contributed nothing, and pregnancy went to term. Delivery was spontaneous and neonatal condition normal.

The optic fundi appeared normal, there was no paresis, the tendon reflexes were present and symmetrically equal, and the plantar responses downgoing. There were no signs of focal neurological damage, and general examination was negative. The child was afebrile throughout. A blood count, sedimentation rate, Mantoux $(1 / 1,000)$ test, chest and skull radiographs, blood lead estimation, and lumbar puncture all revealed no abnormality. A series of electroencephalograms was within normal limits.

Polyelectromyograms taken from the muscles of both upper arms (Dr. Pampiglione) revealed the irregular incidence of single muscle action potentials. No tendency towards synergy of muscular contraction was found.

Three weeks after admission the prednisolone dosage was increased to $20 \mathrm{mg}$. per day, whereupon the intrusion of involuntary movements became less marked. Two weeks later the level was reduced to $10 \mathrm{mg}$. per day, and the disorder regained its initial severity. High dosage of prednisolone was therefore resumed, with unequivocal success. When the dosage was then gradually reduced, 
relapse followed as before, and the sequence of high dosage (30 to $50 \mathrm{mg}$. daily) causing remission and low dosage (less than $15 \mathrm{mg}$. daily) resulting in relapse was repeated a third time. Treatment was then changed to A.C.T.H. units whereupon the involuntary movements ceased altogether. Over the next six weeks it was possible to reduce and finally to stop the A.C.T.H. without recurrence, but two weeks later jerking again became evident. It was finally found that the minimum dose for suppression of symptoms was 12.5 units of A.C.T.H., and the child was sent out on this. At discharge, he was alert and interested, was speaking fairly clearly again, and making good progress in walking. There was now no abnormality on examination. Nine months had elapsed since the onset of the disease. At present, one year after discharge, he remains symptom free and normal development is being resumed, but it has not yet proved possible to withdraw A.C.T.H. treatment, as this results in recurrence.

The clinical appearances in Case 3 were very similar to those in the two previously described patients. Unlike the latter, however, his condition had its onset 10 days after an injection of triple vaccine. Whether the injection was causally related to the motor disorder remains uncertain. The response to steroid and A.C.T.H. treatment, above a 'critical' level, was unusually clear-cut and reproducible in this case.

The next patient similarly failed to repeat an initial good response to prednisone, but then went into complete remission on A.C.T.H.

CASE 4 J.L., aged 3 years, was admitted under the care of Dr. P. H. Sandifer. When aged 18 months, in the course of a mild illness characterized by vomiting and diarrhoea, he began to scream for no apparent reason, and was found to have become unsteady on his feet. His limbs were subject to irregular jerkings, while his eyeballs made 'flickering' movements. He was admitted to the Alder Hey Children's Hospital under the care of Dr. F. P. Hudson, at which time he was unable even to sit up. But apart from the motor disorder no other abnormality was found. A white cell count, Mantoux $(1 / 1,000)$ test, and cerebrospinal fluid examination were negative, as were skull radiographs and dye test for toxoplasma. But complement-fixation tests gave evidence of recent infection with lymphocytic choriomeningitis virus. Electroencephalograms were within normal limits.

Over the next year the degree of disability fluctuated but there was no overall trend towards improvement or deterioration. At times he could walk quite efficiently; at others he was reduced to shuffling across the floor on his bottom. His hand movements remained incoordinate, and his eyes continued to 'flicker' to a variable extent. However, his mental development progressed, and his speech gained in clarity and content. At the age of 20 months he had an exacerbation, and was given prednisolone, $5 \mathrm{mg}$. daily. Over the next two weeks there was a considerable reduction in the motor disorder, but this relapsed to some extent when the drug was gradually withdrawn. Re-introduction of prednisolone then no longer had the same striking effect.

On admission he was a well-nourished, alert boy whose movements were grossly incoordinate. He was unable to walk, but could crawl rapidly across the floor. In the sitting position, violent titubation was apparent, and his hands shook rapidly and irregularly as he handled toys. His eyeballs made rapid conjugate movements of an unpredictable kind, sometimes so far upwards that the corneae would momentarily vanish from sight. His speech, though not unintelligible, was jerky and incoordinate. At rest, scattered muscular contractions could still be observed, particularly around the mouth.

The family history contributed nothing. Normal pregnancy was followed by spontaneous delivery at term; the neonatal condition was normal, as was motor development up to the onset of the motor disorder. There was no abnormality of skull shape and size. The neck was supple. There were no focal neurological signs. The electroencephalogram was repeatedly within normal limits. Laboratory investigations were all negative.

The jerky movements of the limbs were examined by polyelectromyographic recording (Dr. Pampiglione). This revealed movements which were asynergic, and isolated single muscle potentials were detected, though more frequently they were aggregated in short bursts.

The dosage of prednisolone was raised to $40 \mathrm{mg}$. a day but no improvement was observed. Ten days later A.C.T.H. (40 units a day) was introduced, while prednisolone was gradually withdrawn. Within a few days the intrusion of involuntary movements was perceptibly less, and within two weeks it was abolished. When the A.C.T.H. was then completely withdrawn in the course of the next four weeks, there was no recurrence of motor disability. On discharge there were no abnormal physical signs, and the child was plucking up courage to walk freely, and even to run. At the present time, nine months after discharge, he remains well.

This case, though similar to the previous ones in its acute onset, long-drawn out, fluctuating course, and response to therapy, never quite matched them in severity. In the next case to be described, the mildest of the series, the initial response to A.C.T.H. was particularly dramatic.

CASE 5 S.H., aged 10 months, was admitted under the care of Dr. P. H. Sandifer. Four months earlier, during a mild illness characterized by fever and rash, there had been the sudden onset of flickering movements of the eyes and shakiness of the arms. Up to then, she had developed in a normal manner. The flickering and shakiness continued at a constant level of intensity up to the age of 9 months, when one week after poliomyelitis vaccination, the condition became still more marked, and remained so till admission. On admission S.H. appeared to be a well-nourished baby, who showed interest in the outside world, but whose attempts to follow visual lutes were vitiated by the continual intervention of brief, rapid, conjugate movements of the eyeballs, irregular in incidence and unpredictable in direction. Attempts to 
reach for objects were unsuccessful on account of shocklike perturbation of the muscles of the arms, and violent jerking of muscles of the trunk and neck made it impossible for her to maintain a posture. But though most apparent during voluntary movement the jerking was seen to persist at rest, and even to some extent in light sleep. The general picture was of an eager child whose every movement was nullified by a background of uncontrolled muscular activity.

In the family history the only possibly relevant episode was an encephalitic condition which has caused permanent disability in S.H.'s father; details of this are given below.

S.H. was the product of an uneventful twin pregnancy which went to term, and spontaneously resulted in a natural vertex delivery of S.H. as the second of twins, of whom the first died after a breech delivery. By the age of 6 months, when the present condition began, she was following with her eyes, reaching for objects with her hands, and had head control from the prone position.

The skull circumference was $17 \frac{1}{2}$ in., the anterior fontanelle was open and flat, the neck was supple. There was a left concomitant strabismus, but the eye grounds were normal, and there were no focal neurological signs. She was and remained afebrile. A blood count, the sedimentation rate, fasting blood sugar, and blood electrolytes were at normal levels; urinary amino-acid content, a radiograph of the skull, electrocardiogram, electroencephalogram, and pressure and composition of cerebrospinal fluid were all within normal limits.

After a single injection of A.C.T.H. (20 units) the motor disorder disappeared. Treatment at the level of 40 units per day was continued for two weeks, then withdrawn over a period of four weeks, without recurrence. During this period she showed a peculiar tendency to cover her eyes with her hands, not only in bright light, but she would also sleep in this position. A week after treatment was completely withdrawn and she was discharged, the condition recurred, though less severely than before. On re-admission there was again occasional flickering of the eyes, though now not more than two to three times a minute and there was again widespread irregular jetking of muscles. A tendency towards repetitive head movements was also apparent on this occasion.

Investigation again showed a normal blood count and chemistry and cerebrospinal fluid under normal pressure and of normal constitution. Electroencephalography remained non-contributory but polyelectromyography of limb muscles (Dr. Pampiglione) showed the occasional incidence of single muscle potentials as well as short bursts of potentials lacking synergy. Stimulation with A.C.T.H. resulted in the production of urinary steroids in amounts abnormally low for the age. Serum electrophoresis was remarkable only for the presence of an excessive amount of gamma globulin. Virological studies revealed no abnormality.

Reinstitution of A.C.T.H. (40 units per day) again caused disappearance of the muscular jerks. This time, however, there persisted a very slight degree of fine rapid oscillation of the head and trunk, when, as she now could, she maintained the seated position. After withdrawal of A.C.T.H. therapy the recovery was maintained for only one week, but when put on a maintenance dose of 10 units daily, she obtained complete symptomatic relief.

W.H., the father of S.H., aged 37 years, a lorry driver's mate, became subject to recurrent nausea and vomiting and two months later, to severe continuous headaches. Three days later he became dysarthric and developed nystagmus and severe cerebellar ataxia. After a week, he became jaundiced. At the same time the nausea and vomiting receded. A few days later the headaches ceased but the jaundice remained evident for three weeks. Two lumbar punctures yielded cerebrospinal fluid under normal pressure, but showing pleocytosis and a raised protein content.

Severe static and action tremor of cerebellar type has remained present ever since.

W.H.'s condition differed in essential respects from the myoclonic status that affected his daughter 10 months later. The length of this interval makes it difficult to suppose that an infective agent common to both was involved.

CASE 6 K.E., aged 16 months, was admitted under the care of Dr. P. H. Sandifer, having three months earlier become subject to jerking of the eyes and limbs. She lost the ability to stand and to speak, but not her interest in the outside world. On examination she was an alert child who wobbled perilously when maintaining the sitting position, while her head was subject to gross irregula titubation. Her eyes darted rapidly from point to poin $\frac{P}{1}$ in unpredictable movements of deviation and rotation When deviation was in an upward direction, it was associated with rapid, jerky elevation of the eyelids. Af times, the frontalis muscle would jerk on its own. Whe she tried to grasp, her efforts were handicapped by the intrusion of violent muscular jerking.

The family history contributed nothing. There was o toxaemia and antepartum haemorrhage during pregna ncy, and delivery, near term, was by forceps. It proved necessary to administer oxygen on delivery. Nevertheless, she passed her motor milestones at the normal rate, and by the age of 1 year had a vocabulary of more than six words. There was no previous disease of apparent relevance to the motor disorder.

The skull was normal in size and shape. The fundi were unremarkable and there were no focal neurological abnormalities. She was afebrile. A blood count and Mantoux (1/1000) test were negative; liver function tests? gave a normal result, and the urine contained no abnormal amino-acids. The blood test was within the normal range, $\frac{\vec{\partial}}{0}$ and the cerebrospinal fluid was under normal pressure, and of normal composition. An electrocardiogram and 3 an electroencephalogram were within the normal limits.

Polyelectromyography (Dr. Pampiglione) of limb muscles revealed the asynergic incidence of brief bursts of muscle action potentials, as well as isolated potentials. 을

Before admission she had been given prednisone $\left(30_{D}\right.$ mg. a day) with a good result, lasting, however, only a week. She was therefore started on A.C.T.H. (40 units a day) and the involuntary movements decreased greatly $\Omega$ within a week. Treatment with A.C.T.H. is being con- N tinued. \begin{tabular}{l}
\hline \\
\hline \\
\hline
\end{tabular} 
The salient features of the motor disorder which affected the patients of this series may now be summarized.

The six children (three boys, three girls) aged 9 to 20 months at the onset of the disease, had all, with the exception of Case 1, developed normally up to that time. No definite precipitating factor preceded the onset in Cases 5 and 6. The onset in a setting of upper respiratory tract infection in Cases 1 and 2, of gastrointestinal infection in Case 4, and following an injection of triple vaccine in Case 3 is of uncertain significance. In the course of a week or less a disorder characterized by the frequent, irregular and widespread intrusion of shock-like muscular contractions had reached maximum severity. The appearances were of an irregular and variable incoordination of limb movements, irregular titubation, and flickering muscular contractions of unpredictable distribution at rest. The eyeballs were notably affected by rapid and irregular conjugate movements of displacement and rotation shown by cine recordings to occur at up to eight a second. The flickering muscular contractions and 'dancing eyes' produced an unmistakable clinical appearance. They were at a maximum when the child was startled, in abeyance only during deep sleep, unaffected by anticonvulsant therapy, and unassociated with discharges on the electroencephalogram. There were no other abnormalities on neurological or general examination. Mental clouding was not a feature. Investigations revealed no evidence of systemic disorder, and skull radiographs were negative. Cerebrospinal fluid examination was consistently negative as regards pressure, cell, protein and sugar content, and the Lange reaction. In Case 1 repeated air studies and cortical biopsy were within normal limits. No epileptic phenomena preceded the onset or occurred during the disease. Electroencephalograms were at all times within normal limits. Polyelectromyographic recordings from the limb muscles were made in Cases 3, 4, 5, and 6 and single muscle potentials occurring irregularly in unpredictable distribution as well as brief bursts of asynergic muscle action potentials were detected. The course of the disease was prolonged, fluctuating, but even over a period of years, not progressive. Treatment with A.C.T.H. succeeded in abolishing symptoms in all cases in which it was tried (Cases 3, 4, and 5), the recovery being maintained when the drug was gradually withdrawn in Case 4. In Case 3 and 5 a maintenance dose of A.C.T.H. is at present still necessary. Case 6 is responding well to A.C.T.H.

\section{DISCUSSION}

The six patients described here were subject to a motor disorder of highly distinctive type. Its salient features, nearly identical in every case, differed in essential respects from those of the recognized causes of incoordination in children.

Cerebellar syndromes characterized by static or action tremor, such as 'acute cerebellar ataxia', can readily be excluded. The rhythmic regularity and predictable incidence of cerebellar tremor was absent. At times, a movement was disorganized from its inception; at other times, it was completed smoothly and without perturbation. Similarly, the irregular and unpredictable incidence of jerking would determine whether a posture could be even briefly maintained or not. The chaotic irregularity of the involuntary eye movements distinguished them from the phasic nystagmus of some cases of acute cerebellar ataxia, the opsoclonia found on occasion in non-epidemic encephalitis (Orzechowski, 1927) and polioencephalitis (Marmion and Sandilands, 1947), and the 'cog-wheel' rotary nystagmus of the Pelizaeus-Merzbacher syndrome. The irregularity and distribution of the involuntary movements, which affect the limbs as well as the eyes and axial musculature, differentiates the condition from spasmus nutans (Østerberg, 1937).

Myoclonus may simulate an action tremor in that it is accentuated during active movement (Dawson, 1947). This occurred in the present cases. But the persistence of a less marked degree of irregular jerking at rest and even during sleep was clinical evidence of the presence of myoclonus. The asynergic character of the involuntary movements, an essential feature of myoclonus (Bogaert, Radermecker, and Titeca, 1950), was established by polyelectromyography (Pampiglione, 1959).

Once the myoclonic nature of the motor disorder is recognized, the problem becomes that of the differential diagnosis of myoclonic status. Where myoclonic status occurs in idiopathic epilepsy, familial myoclonic epilepsy (Unverricht, 1895), the Ramsey Hunt syndrome (Hunt, 1921), or cerebral lipoidosis (Gastaut and Rémond, 1952; Cobb, Martin, and Pampiglione, 1952) it is associated with a cerebral dysrhythmia reflected in the electroencephalogram. In the present cases the E.E.G.s were consistently within normal limits. The anatomical factors that account for the failure of 'upstream' discharge to the cerebral cortex to occur are as yet unknown; but the absence of E.E.G. abnormalities is an important diagnostic feature.

The course of the disorder is no less characteristic. The myoclonic status is not shortlived and responsive to anticonvulsants as in idiopathic epilepsy, permanent, as it may be following encephalitis lethargica (Lennox, 1960), or insidiously progressive, as in the degenerative conditions mentioned above. Its acute 
onset, protracted but non-progressive course, and ultimate spontaneous remission distinguish it from all other myoclonic syndromes. The dramatic response to steroid therapy, and particularly to A.C.T.H., is a further distinguishing feature. The action of these drugs as reported in the acute postinfectious encephalitides (Hartmann, 1957; Öberg, 1959 ) is dubious, as remission might well have been coincidental, as is suggested by a controlled study (Ziegra, 1961). In the present cases their beneficial effect was demonstrated beyond doubt. Not only was remission rapid even when the disorder was already of long standing, but withdrawal of therapy (on four occasions in Case 3 and in two in Case 5) was regularly followed by relapse. In Cases 3 and 5 it was even possible to establish with fair accuracy the minimum maintenance dose of A.C.T.H. sufficient to suppress the symptoms.

The mechanism of the action of steroids in these cases, and the reasons why A.C.T.H. is so especially effective, are unknown. If the myoclonic encephalopathy is an allergic manifestation, as seems not unlikely, it may be the anti-allergic action of steroids which gives symptomatic relief. A reasonable guess might ke that in these children nonspecific causes (virus infections, immunization, etc.) have set up a self-perpetuating auto-immune process lasting for years, and uncovered every time A.C.T.H. administraticn falls below a critical minimum level.

The disorder is remarkably limited in its extent and functional consequences. Myoclonus is the only demonstrable abnormality. Mental clouding is conspicuously absent, and once steroid therapy has secured symptomatic relief from the myoclonus, the acquisition of motor skills is resumed in the normal way and lost ground gradually made good. Cerebral cortical function is unaffected. The encephalopathy is presumably at brain-stem (and perhaps spinal cord) level. But myoclonus has been claimed to arise from a wide variety of brain-stem structures (Greenfield, 1958) and in the absence of other physical signs referable to the brain-stem, and of necropsy material, no more accurate localization can be attempted.

An estimate of the frequency of myoclonic encephalopathy of infants will have to await the publication of further case reports from other centres. In the past, such cases may have been unrecognized and included in series of 'acute cerebellar ataxia'. This may in particular be true of one or two cases in the series of Cottom (1957) and of Walcher and Ross (1958). The child reported by
Keller and Karelitz (1948), whose disease began at the age of 18 months, showed a motor disorder that might well be interpreted as myoclonic.

The diagnosis is nevertheless not a difficult one, and can be made with confidence on clinical grounds alone, although E.E.G.s and polyelectromyograms (Pampiglione, 1959) give useful supporting evidence. Laboratory investigations, including the examination of the cerebrospinal fluid, have proved noncontributory, except in a negative sense. Increasing familiarity with the condition has made it possible to dispense with the more drastic procedures of air encephalography, ventriculography, and cortical biopsy. Effective symptomatic treatment is available, and the experience outlined here makes it possible to give a good long-term prognosis even in the face of a motor disorder of the utmost severity.

\section{SUMMARY}

Six cases are presented of a hitherto undescribed condition characterized by the acute onset, in infancy, of incoordination due to the frequent, widespread, and irregular intrusion of myoclonic jerking as an isolated disorder. The condition pursued a protracted but non-progressive and ultimately self-limiting course. Striking symptomatic relief was derived from treatment with A.C.T.H.

I wish to thank Dr. P. R. Evans, Dr. R. S. Lightwood, and Dr. P. H. Sandifer for permission to report on patients under their care. I am especially indebted to Dr. P. H. Sandifer for suggesting this work, and for? generous advice and encouragement.

\section{REFERENCES}

Bogaert, L. van, Radermecker, J., and Titeca, J. (1950). Folia psychiat. neerl., 53, 650 .

Cobb, W., Martin, F., and Pampiglione, G. (1952). Brain, 75, 343.

Cottom, D. G. (1957). Arch. Dis. Childh., 32, 181.

Dawson, G. D. (1947). J. Neurol. Neurosurg. Psychiat., 10, 141.

Gastaut, H., and Rémond, A. (1952). Rev. neurol., 86, 596.

Greenfield, J. G. (1958). Neuropathology. Arnold, London.

Hartmann, H. (1957). Arztl. Wschr., 12, 533.

Hunt, J. R. (1921). Brain, 44, 490.

Keller, M. J., and Karelitz, S. (1948). Pediatrics, 1, 754.

Lennox, W. G. (1960). Epilepsy and Related Disorders. Churchill, London.

Marmion, D. E., and Sandilands, J. (1947). Lancet, 2, 508.

Oberg, G. (1959). Acta paediat. (Uppsala), 48, Suppl. 117, p. 56.

Orzechowski, C. (1927). J. Psychol. Neurol. (Lpz.), 35, 1.

Østerberg, G. (1937). Acta Ophthal., 15, 457.

Pampiglione, G. (1959). Cerebral Palsy Bull., 1, no. 7, p. 10.

Unverricht, M. (1895). Dtsch. Z. Nervenheilk., 7, 32.

Walcher, D. N., and Ross, A. T. (1958). A.M.A. J. Dis. Child., 96, 278.

Ziegra, S. R. (1961). J. Pediat., 59, 322. 